\title{
Analytical and Numerical Solution of 2D Problem for Transversely Isotropic Generalized Thermoelastic Medium with Green-Naghdi Model II
}

\author{
Ibrahim A. Abbas \\ Department of Mathematics, Faculty of Science, Sohag University, Sohag, Egypt. \\ Nonlinear Analysis and Applied Mathematics Research Group (NAAM), Department of Mathematics, King Abdu- \\ laziz University, Jeddah, Saudi Arabia.
}

\author{
Mohamed I. A. Othman \\ Department of Mathematics, Faculty of Science, Zagazig University, Egypt. \\ Department of Mathematics, Faculty of Science, Taif University 888, Taif, Saudi Arabia.
}

\begin{abstract}
(Received 10 March 2015; accepted 10 June 2016)
In this paper, a comparison was made between the analytical and numerical solution of a two-dimensional problem for a transversely isotropic generalized thermoelastic medium. The study is carried out in the context of generalized thermoelasticity proposed by Green and Naghdi's theory of type II. The problem has been solved analytically using the normal mode method with the eigenvalue approach and numerically using a finite element method. The accuracy of the finite element formulation was validated by comparing the analytical and numerical solutions for the field quantities.
\end{abstract}

\section{INTRODUCTION}

Thermoelasticity theories, which admit a finite speed for thermal signals (second sound), have aroused much interest in the last three decades. In contrast to the conventional coupled thermoelasticity theory based on a parabolic heat equation, ${ }^{1-3}$ which predicts an infinite speed for the propagation of heat, these theories involve a hyperbolic heat equation and are referred to as generalized thermoelasticity theories. Among these generalized theories, the theory proposed by Lord and Shulman ${ }^{4}$ involving one relaxation time and the theory developed by Green and Lindsay ${ }^{5}$ involving two relaxation times have been subjected to a large number of investigations. In the Lord-Shulman theory, a modified Fourier's law of heat conduction including both the heat and its time derivative replaces the conventional Fourier's law whereas in the Green-Lindsay model, Fourier's law of heat conduction is left unchanged but the classical energy equation and stress-strain temperature relations are modified. Verma and Hasebe ${ }^{6}$ studied wave propagation in transversely isotropic plates in the context of generalized thermoelasticity proposed by the Lord-Shulman theory. Othman ${ }^{7}$ studied the dependence of the modulus of elasticity on the reference temperature in a two-dimensional problem of generalized thermoelasticity under Lord-Shulman theory. In view of some experimental evidence available in favour of finiteness of heat propagation speed, generalized thermoelasticity theories are considered to be more realistic than the conventional thermoelasticity theory in dealing with practical problems involving very large heat fluxes and short time inter- vals, like those occurring in laser units and energy channels. The relevant literature can be found in Chandrasekharaiah, ${ }^{8}$ Othman, ${ }^{9-11}$ and Ignaczak. ${ }^{12}$

On the experimental side, available evidence in support of the existence of finite thermal wave speed in solids is rather sparse, although an experimental study for second sound propagation in dielectric solids and some related experimental observations were reported nearly four decades ago. ${ }^{13-18}$ Most engineering materials such as metals possess a relatively high rate of thermal damping and thus are not suitable for use in experiments concerning second sound propagation. But, given the state of recent advances in material science, it may be possible in the foreseeable future to identify (or even manufacture for laboratory purposes) an idealized material for the purpose of studying the propagation of thermal waves at finite speed.

Relevant theoretical developments on the subject were made by Green and Naghdi. ${ }^{19}$ They developed three models for generalized thermoelasticity of homogeneous isotropic materials which are labelled as model I, II, and III. The nature of these theories is such that when the respective theories are linearized, model $\mathrm{I}^{20}$ reduces to the classical heat conduction theory based on Fourier's law. The linearized versions of model II and III permit propagation of thermal waves at finite speed. Model II, in particular, exhibits a feature that is not present in the other established thermoelastic models as it does not sustain dissipation of thermal energy. ${ }^{20}$ In this model, the constitutive equations are derived by starting with the reduced energy equation and by including the thermal displacement gradient among other constitutive variables. Green-Naghdi's third model ${ }^{21}$ ad- 
mits the dissipation of energy. In this model, the constitutive equations are derived by starting with the reduced energy equation, where the thermal displacement gradient in addition to temperature gradient are among the constitutive variables. In the context of the linearized version of this theory, ${ }^{20}$ the theorem on uniqueness of solutions has been established by Chandrasekharaiah. ${ }^{22,23}$ Othman and Atwa ${ }^{24}$ studied the effect of magnetic fields on a two-dimensional problem of generalized thermoelasticity without energy dissipation. Othman and Atwa ${ }^{25}$ have also studied the response of micropolar thermoelastic medium with voids due to various sources with and without energy dissipation. The problems have been solved by applying the eigenvalue approach. Roychoudhuri and Dutta ${ }^{26}$ studied thermoelastic interactions in an isotropic homogeneous thermoelastic solid containing time-dependent distributed heat sources which vary periodically for a finite time interval in the context of TEWOED. Problems concerning generalized thermoelasticity proposed by Green and Naghdi's theory of both types II and III have been studied by many other authors. ${ }^{27,28}$

The exact solution of the generalized thermoelasticity theory governing equations for a coupled and non-linear/linear exists only for very special and simple initial and boundary problems. In view of calculating general problems, a numerical solution technique is to be used. For this reason, the finite element method (FEM) is chosen. The method of weighted residuals offers us the formulation of the finite element equations and we obtain best approximated solutions to linear and nonlinear ordinary and partial differential equations. Applying this method basically involves three steps. The first step is to assume the general behavior of the unknown field variables in such a way as to satisfy the given differential equations. Substitution of these approximating functions into the differential equations and boundary conditions results in some errors, called the residual. This residual has to vanish in an average sense over the solution domain. The second step is the time integration. The time derivatives of the unknown variables have to be determined by former results. The third step is to solve the equations resulting from the first and the second step using the solving algorithm of the finite element program. ${ }^{29}$ Abbas and his colleagues ${ }^{30-39}$ applied the FEM in different problems.

In the present paper, we have formulated the problem of a two-dimensional problem for a transversely isotropic generalized thermoelasticity under the Green-Naghdi theory of type II. The problem has been solved analytically using a normal mode method with the eigenvalue approach and numerically using a FEM. The results for a transversely isotropic material have been deduced numerically and presented graphically to compare with those of transversely isotropic material which was obtained analytically.

\section{FORMULATION OF THE PROBLEM}

We consider the problem of a transversely isotropic thermoelastic half-space $(x \geq 0)$. The surface of the half-space is subjected to a thermal shock, which is a function of $y$ and $t$. Thus, all the quantities considered will be functions of the time variable $t$, and of the coordinates $x$ and $y$. Then the displacement vector $\mathbf{u}$ and temperature $T$ can be taken in the following form

$$
\mathbf{u}=(u(x, y, t), v(x, y, t), 0), \quad T=T(x, y, t) .
$$

Now the constitutive relations in the present case are

$$
\begin{aligned}
\sigma_{x x} & =C_{11} e_{x x}+C_{12} e_{y y}-\beta_{11}\left(T-T_{0}\right) ; \\
\sigma_{y y} & =C_{12} e_{x x}+C_{11} e_{y y}-\beta_{11}\left(T-T_{0}\right) ; \\
\sigma_{x y} & =\left(C_{11}-C_{12}\right) e_{x y} ;
\end{aligned}
$$

The equations of motion along $x$ and $y$ directions can be obtained as follows

$$
\begin{aligned}
\frac{\partial \sigma_{x x}}{\partial x}+\frac{\partial \sigma_{x y}}{\partial y} & =\rho \frac{\partial^{2} u}{\partial t^{2}} ; \\
\frac{\partial \sigma_{y x}}{\partial x}+\frac{\partial \sigma_{y y}}{\partial y} & =\rho \frac{\partial^{2} v}{\partial t^{2}} ; \\
K^{*}\left(\frac{\partial^{2} T}{\partial x^{2}}+\frac{\partial^{2} T}{\partial y^{2}}\right) & =\rho C_{E} \frac{\partial^{2} T}{\partial t^{2}}+\beta_{11} T_{0} \frac{\partial^{2} e}{\partial t^{2}} ;
\end{aligned}
$$

or,

$$
\begin{gathered}
C_{11} \frac{\partial^{2} u}{\partial x^{2}}+\frac{C_{11}-C_{12}}{2} \frac{\partial^{2} u}{\partial y^{2}}+ \\
\frac{C_{11}+C_{12}}{2} \frac{\partial^{2} v}{\partial x \partial y}-\beta_{11} \frac{\partial T}{\partial x}=\rho \frac{\partial^{2} u}{\partial t^{2}} \\
\frac{C_{11}-C_{12}}{2} \frac{\partial^{2} v}{\partial x^{2}}+C_{11} \frac{\partial^{2} v}{\partial y^{2}}+ \\
\frac{C_{11}+C_{12}}{2} \frac{\partial^{2} u}{\partial x \partial y}-\beta_{11} \frac{\partial T}{\partial y}=\rho \frac{\partial^{2} v}{\partial t^{2}} ; \\
K^{*}\left(\frac{\partial^{2} T}{\partial x^{2}}+\frac{\partial^{2} T}{\partial y^{2}}\right)=\rho C_{E} \ddot{T}+\beta_{11} T_{0}\left(\frac{\partial \ddot{u}}{\partial x}+\frac{\partial \ddot{v}}{\partial y}\right) ;
\end{gathered}
$$

where $e=\frac{\partial u}{\partial x}+\frac{\partial v}{\partial y}, e_{x x}=\frac{\partial u}{\partial x}, e_{y y}=\frac{\partial v}{\partial x}, e_{x y}=\frac{\partial u}{\partial y}+\frac{\partial v}{\partial x}$, $\beta_{11}$ is the stress temperature coefficient, $C_{E}$ is the specific heat at constant volume, and $K^{*}$ is the additional material constant for Green-Naghdi theory of type II.

The following nondimensional variables are introduced:

$$
\begin{array}{rlrl}
\left(x^{\prime}, y^{\prime}\right) & =\frac{1}{L}(x, y), & t^{\prime} & =\frac{c_{1}}{L} t, \\
\left(u^{\prime}, v^{\prime}\right) & =\frac{C_{11}}{L \beta_{11} T_{0}}(u, v), & T^{\prime} & =\frac{\beta_{11}\left(T-T_{0}\right)}{C_{11}}, \\
\left(\sigma_{x x}^{\prime}, \sigma_{x y}^{\prime}, \sigma_{x y}^{\prime}\right) & =\frac{1}{\beta_{11} T_{0}}\left(\sigma_{x x}, \sigma_{x y}, \sigma_{x y}\right), & c_{1}^{2}=\frac{C_{11}}{\rho} ;(11)
\end{array}
$$

and in terms of the non-dimensional quantities defined in Eq. (11), the above governing equations reduce to (dropping the dashed for convenience)

$$
\begin{aligned}
\sigma_{x x} & =\frac{\partial u}{\partial x}+a_{1} \frac{\partial v}{\partial y}-T ; \\
\sigma_{y y} & =a_{1} \frac{\partial u}{\partial x}+\frac{\partial v}{\partial y}-T ; \\
\sigma_{x y} & =a_{2}\left(\frac{\partial v}{\partial x}+\frac{\partial u}{\partial y}\right) ; \\
\frac{\partial \sigma_{x y}}{\partial x}+\frac{\partial \sigma_{x y}}{\partial y} & =\frac{\partial^{2} u}{\partial x} ; \\
\frac{\partial \sigma_{x y}}{\partial x}+\frac{\partial \sigma_{y y}}{\partial y} & =\frac{\partial^{2} v}{\partial t^{2}} ; \\
\varepsilon_{1}\left(\frac{\partial^{2} T}{\partial x^{2}}+\frac{\partial^{2} T}{\partial y^{2}}\right) & =\frac{\partial^{2} T}{\partial t^{2}}+\varepsilon_{2} \frac{\partial^{2} e}{\partial t^{2}}
\end{aligned}
$$


where $a_{1}=\frac{C_{12}}{C_{11}}, a_{2}=\frac{C_{11}-C_{12}}{C_{11}}=\left(1-a_{1}\right), \varepsilon_{1}=\frac{K^{*}}{\rho C_{E} C_{1}^{2}}$, $\varepsilon_{2}=\frac{\beta_{11}^{2} T_{0}}{\rho C_{E} C_{11}}$.

\section{NORMAL MODE ANALYSIS}

The solution of the considered physical variable can be decomposed in terms of normal modes as the following form:

$$
\left[u, v, T, \sigma_{i j}\right](x, y, t)=\left[u^{*}, v^{*}, T^{*}, \sigma_{i j}^{*}\right](x) \exp (\omega t+i b y)
$$

where $\omega$ is a complex constant, $i=\sqrt{-1}, b$ is the wave number in the $y$ direction and $u^{*}(x), v^{*}(x), T^{*}(x)$ and $\sigma_{i j}^{*}(x)$ are the amplitudes of the field quantities. Subsisting from Eq. (18) in Eqs. (12)-(17), we get

$$
\begin{aligned}
\sigma_{x x}^{*} & =\frac{d u^{*}}{d x}+i b a_{1} v^{*}-T^{*} ; \\
\sigma_{y y}^{*} & =a_{1} \frac{d u^{*}}{d x}+i b v^{*}-T^{*} ; \\
\sigma_{x y}^{*} & =a_{2}\left(i b u^{*}+\frac{d v^{*}}{d x}\right) ; \\
e^{*} & =\frac{d u^{*}}{d x}+i b v^{*} ; \\
\frac{d^{2} u^{*}}{d x^{2}} & =B_{41} u^{*}+B_{45} \frac{d v^{*}}{d x}+B_{46} \frac{d T^{*}}{d x} \\
\frac{d^{2} v^{*}}{d x^{2}} & =B_{52} v^{*}+B_{53} T^{*}+B_{54} \frac{d u^{*}}{d x} \\
\frac{d^{2} T^{*}}{d x^{2}} & =B_{62} v^{*}+B_{63} T^{*}+B_{64} \frac{d u^{*}}{d x}
\end{aligned}
$$

where $B_{41}=b^{2} a_{2}+\omega^{2}, B_{45}=-i b, B_{46}=1, B_{52}=\frac{1}{a_{2}}\left(b^{2}+\right.$ $\left.\omega^{2}\right), B_{53}=\frac{i b}{a_{2}}, B_{54}=-\frac{i b}{a_{2}}, B_{62}=\frac{i b \varepsilon_{2} \omega_{2}}{\varepsilon_{1}}, B_{63}=b^{2}+\frac{\omega^{2}}{\varepsilon_{1}}$, $B_{64}=\frac{\varepsilon_{2} \omega^{2}}{\varepsilon_{1}}$. Equations (23)-(25) can be written in a vectormatrix differential equation as follows: $:^{40-43}$

$$
\frac{d \vec{V}}{d x}=\mathbf{B} \vec{V}
$$

where $\vec{V}=\left[\begin{array}{llllll}u^{*} & v^{*} & T^{*} & \frac{d u^{*}}{d x} & \frac{d v^{*}}{d x} & \frac{d T^{*}}{d x}\end{array}\right]^{T}$ and

$$
\mathbf{B}=\left(\begin{array}{cccccc}
0 & 0 & 0 & 1 & 0 & 0 \\
0 & 0 & 0 & 0 & 1 & 0 \\
0 & 0 & 0 & 0 & 1 & 0 \\
B_{41} & 0 & 0 & 0 & B_{45} & B_{46} \\
0 & B_{52} & B_{53} & B_{54} & 0 & 0 \\
0 & B_{62} & B_{63} & B_{64} & 0 & 0
\end{array}\right)
$$

\section{SOLUTION OF THE VECTOR-MATRIX DIFFERENTIAL EQUATION}

Let us now proceed to solve Eq. (26) by the eigenvalue approach proposed. The characteristic equation of the matrix $\mathbf{B}$ takes the form

$$
\lambda^{6}-\eta_{1} \lambda^{4}+\eta_{2} \lambda^{2}+\eta_{3}=0
$$

where $\eta_{1}=B_{41}+B_{52}+B_{45} B_{54}+B_{63}+B_{46} B_{64}, \eta_{2}=$ $B_{41} B_{52}-B_{53} B_{62}-B_{46} B_{54} B_{62}+B_{41} B_{63}+B_{52} B_{63}+$ $B_{45} B_{54} B_{63}+B_{46} B_{52} B_{64}-B_{45} B_{53} B_{64}, \eta_{3}=B_{41} B_{53} B_{62}-$ $B_{41} B_{52} B_{63}$. The roots of the characteristic Eq. (27), which are also the eigenvalues of matrix $\mathbf{B}$, are of the form $\lambda=$ $\pm \lambda_{1}, \lambda= \pm \lambda_{2}, \lambda= \pm \lambda_{3}$. The eigenvector $\vec{X}=$ $\left[x_{1}, x_{2}, x_{3}, x_{4}, x_{5}, x_{6}\right]^{T}$, corresponding to eigenvalue $\lambda$, can be calculated as

$$
\begin{aligned}
& x_{1}=-\lambda\left(B_{45} B_{53}+B_{46}\left(-B_{52}+\lambda^{2}\right)\right) ; \\
& x_{2}=B_{41} B_{53}-\left(B_{53}+B_{46} B_{54}\right) \lambda^{2} ; \\
& x_{3}=-B_{41} B_{52}+\left(B_{41}+B_{52}+B_{45} B_{54}\right) \lambda^{2}-\lambda^{4} ; \\
& x_{4}=\lambda x_{1} ; \quad x_{5}=\lambda x_{2} ; \quad x_{6}=\lambda x_{3} .
\end{aligned}
$$

From Eqs. (28)-(31) we can easily calculate the eigenvector $\vec{X}_{j}$, corresponding to eigenvalue $\lambda_{j}, j=1,2,3,4,5,6$. For further reference, we shall use the following notations:

$$
\begin{aligned}
& \vec{X}_{1}=[\vec{X}]_{\lambda=-\lambda_{1}}, \quad \vec{X}_{2}=[\vec{X}]_{\lambda=-\lambda_{2}}, \quad \vec{X}_{3}=[\vec{X}]_{\lambda=-\lambda_{3}}, \\
& \vec{X}_{4}=[\vec{X}]_{\lambda=\lambda_{1}}, \quad \vec{X}_{5}=[\vec{X}]_{\lambda=\lambda_{2}}, \quad \vec{X}_{6}=[\vec{X}]_{\lambda=\lambda_{3}} .
\end{aligned}
$$

The solution of Eq. (26) can be written as follows:

$$
\begin{aligned}
\vec{V} & =\sum_{j=1}^{6} A_{j} \vec{X}_{j} e^{\lambda_{j} x} \\
& =A_{1} \vec{X}_{1} e^{-\lambda_{1} x}+A_{2} \vec{X}_{2} e^{-\lambda_{2} x}+A_{3} \vec{X}_{3} e^{-\lambda_{3} x}
\end{aligned}
$$

where the terms containing exponentials of growing nature in the space variable $x$ have been discarded due to the regularity condition of the solution at infinity, $A_{1}, A_{2}$, and $A_{3}$ are constants to be determined from the boundary condition of the problem. Thus, the field variables can be written for $x \geq 0$, $t>0,-\infty \leq y \leq \infty$ as:

$$
\begin{aligned}
& u(x, y, t)=e^{(\omega t+i b y)} \sum_{j=1}^{3} A_{j} x_{4}^{j} e^{-\lambda_{j} x} \\
& v(x, y, t)=e^{(\omega t+i b y)} \sum_{j=1}^{3} A_{j} x_{5}^{j} e^{-\lambda_{j} x} \\
& T(x, y, t)=e^{(\omega t+i b y)} \sum_{j=1}^{3} A_{j} x_{6}^{j} e^{-\lambda_{j} x}
\end{aligned}
$$

To complete the solution, we have to know the constants $A_{1}$, $A_{2}$, and $A_{3}$, so we will use the following boundary conditions.

\section{APPLICATION}

1. The mechanical boundary condition that the bounding plane to the surface $x=0$ has no traction, so we have

$$
\sigma_{x x}(0, y, t)=\sigma_{x y}(0, y, t)=0 .
$$

2. The thermal boundary condition is

$$
v T-\frac{\partial T}{\partial x}=r(0, y, t)
$$

where $\frac{\partial T}{\partial x}$ denotes the normal components of the heat flux vector, $v$ is the Biot's number, and $r(0, y, t)$ represents the intensity of the applied heat sources. From the boundary conditions Eqs. (37) and (38), we can obtain

$$
\left(\begin{array}{l}
A_{1} \\
A_{2} \\
A_{3}
\end{array}\right)=\left(\begin{array}{lll}
X_{11} & X_{12} & X_{13} \\
X_{21} & X_{22} & X_{23} \\
X_{31} & X_{32} & X_{33}
\end{array}\right)^{-1}\left(\begin{array}{c}
0 \\
0 \\
r^{*}
\end{array}\right) ;
$$


where $X_{11}=-\lambda_{1} x_{4}^{1}+i b a_{1} x_{5}^{1}-x_{6}^{1}, X_{12}=-\lambda_{2} x_{4}^{2}+i b a_{1} x_{5}^{2}-$ $x_{6}^{2}, X_{13}=-\lambda_{3} x_{4}^{3}+i b a_{1} x_{5}^{3}-x_{6}^{3}, X_{21}=i b x_{4}^{1}-\lambda_{1} x_{5}^{1}, X_{22}=$ $i b x_{4}^{2}-\lambda_{2} x_{5}^{2}, X_{23}=i b x_{4}^{3}-\lambda_{3} x_{5}^{3}, X_{31}=\left(v+\lambda_{1}\right) x_{6}^{1}, X_{32}=$ $\left(v+\lambda_{2}\right) x_{6}^{2}, X_{33}=\left(v+\lambda_{3}\right) x_{6}^{3}$.

\section{FINITE ELEMENT METHOD}

The finite element method is a powerful technique originally developed for numerical solutions of complex problems in structural mechanics, and it remains the method of choice for complex systems. A further benefit of this method is that it allows physical effects to be visualized and quantified regardless of experimental limitations. In this section, the governing equations of the two-dimensional problem for transversely isotropic generalized thermoelastic medium without energy dissipation are summarized, using the corresponding finite element equations. In the FEM, the displacement components $u, v$ and temperature $T$ are related to the corresponding nodal values by

$$
u=\sum_{i=1}^{m} N_{i} u_{i}(t), \quad v=\sum_{i=1}^{m} N_{i} v_{i}(t), \quad T=\sum_{i=1}^{m} N_{i} T_{i}(t)
$$

where $m$ denotes the number of nodes per element, and $N$ the shape functions. In the framework of standard Galerkin procedure, the weighting functions and the shape functions coincide. Thus,

$$
\delta u=\sum_{i=1}^{m} N_{i} \delta u_{i}, \quad \delta v=\sum_{i=1}^{m} N_{i} \delta v_{i}, \quad \delta T=\sum_{i=1}^{m} N_{i} \delta T_{i} .
$$

Thus, the finite element equations corresponding to Eqs. (23)(25) can be obtained as

$$
\begin{aligned}
& \int_{0}^{\infty} \frac{d \delta u^{*}}{d x} \frac{d u^{*}}{d x} d x+ \\
& \int_{0}^{\infty} \delta u^{*}\left(B_{41} u^{*}+B_{45} \frac{d v^{*}}{d x}+B_{46} \frac{d T^{*}}{d x}\right) d x=\left.\delta u^{*} \frac{d u^{*}}{d x}\right|_{0} ^{\infty}
\end{aligned}
$$$$
\int_{0}^{\infty} \frac{d \delta v^{*}}{d x} \frac{d v^{*}}{d x} d x+
$$$$
\int_{0}^{\infty} \delta v^{*}\left(B_{52} v^{*}+B_{53} T^{*}+B_{54} \frac{d u^{*}}{d x}\right) d x=\left.\delta v^{*} \frac{d v^{*}}{d x}\right|_{0} ^{\infty} ;
$$

$$
\begin{aligned}
& \int_{0}^{\infty} \frac{d \delta T^{*}}{d x} \frac{d T^{*}}{d x} d x+ \\
& \int_{0}^{\infty} \delta T^{*}\left(B_{62} v^{*}+B_{63} T^{*}+B_{64} \frac{d u^{*}}{d x}\right) d x=\left.\delta T^{*} \frac{d T^{*}}{d x}\right|_{0} ^{\infty} .
\end{aligned}
$$

\section{NUMERICAL RESULTS AND DISCUSSION}

With the view of illustrating the numerical results, the material chosen for the plate is magnesium $(\mathrm{Mg})$, the physical data for which is given by the following: ${ }^{44} C_{11}=5.974 \times$ $10^{10} \mathrm{Nm}^{-2}, C_{12}=2.624 \times 10^{10} \mathrm{Nm}^{-2}, C_{E}=1.04 \times$ $10^{3} \mathrm{Jkg}^{-1} \mathrm{deg}^{-1}, C_{13}=2.17 \times 10^{10} \mathrm{Nm}^{-2}, C_{33}=6.17 \times$ $10^{10} \mathrm{Nm}^{-2}, C_{44}=1.51 \times 10^{10} \mathrm{Nm}^{-2}, \rho=1.74 \times 10^{3} \mathrm{kgm}^{-3}$, $T_{0}=298 \mathrm{~K}, \beta_{11}=2.68 \times 10^{6} \mathrm{Nm}^{-2} \mathrm{deg}^{-1}, \varepsilon_{2}=2.02 \times 10^{-2}$, $K^{*}=200, K_{1}=1.7 \times 10^{2} \mathrm{Wm}^{-1} \mathrm{deg}^{-1}$.

Figures 1-6 are drawn to give a comparison of the results for displacements, temperature, and stresses with that of an isotropic material for two methods of solution under the Green-Naghdi theory of type II, against the thickness $x$ for $y=0.25,1$ and $t=0.2,0.4$. Figures 1 and 2 are plotted to show the variation of displacements $u$ and $v$ against $x$ and make a comparison of the results which obtained by the analytical and numerical solution with isotropic material for the Green-Naghdi model II. The effect of the values of $y$ and $t$ is directly proportional with the value of the thickness $x$ on the distributions for both of the displacement components $u$ and $v$, since the distributions are increasing with the increase of the values of $y$ and $t$ for $x>0$.

Figure 3 clarifies the variation of temperature $T$ against $x$ and study a comparison of the results which were obtained by the analytical and numerical solution with isotropic material for the Green-Naghdi model II. The distribution of $T$ decreases with the increase of $y$ and $t$ values for $x>0$. Figures 4,5 and 6 are plotted to show the variation of stress components $\sigma_{x x}$, $\sigma_{x y}$ and $\sigma_{y y}$ against $x$ and make a comparison of the results which were obtained by the analytical and numerical solution with isotropic material for the Green-Naghdi model II. The distributions of $\sigma_{x x}$ and $\sigma_{y y}$ increase with the increase of $y$ and $t$ values for $x>0$, while the distribution of $\sigma_{x y}$ decreases with the increase of $y$ and $t$ values for $x>0$. It is clear that the qualitative behaviour of the analytical solution and the numerical solution seem to be identical for all comparisons, and all the curves converge to zero as $x \rightarrow \infty$.

The value of the error is directly proportional to the intensity of the applied heat source $r$ for the distributions of both the displacement components $u$ and $v$ and the temperature $T$ where $0 \leq r \leq 4$; the value of error lies between $10^{-12}$ and $10^{-9}$ for the distribution of $u$, the value of error lies between $10^{-11}$ and $10^{-8}$ for the distribution of $v$, and the value of error lies between $10^{-13}$ and $10^{-11}$ for the distribution of $T$. As a result of the very small value of error, two solutions obtained by the normal mode analysis method and the FEM seem to be identical.

Finally, after validating the FEM solutions, one can discuss the following comparison in the case, which has its exact solutions. Table 1 shows a comparison of the FEM solution with the exact solution for displacement $u$. Table 2 shows a comparison of the FEM solution with the exact solution for displacement $v$. Table 3 shows a comparison of the FEM solution with the exact solution for temperature distributions. It is clear from the results that the FEM is more efficient and accurate. 


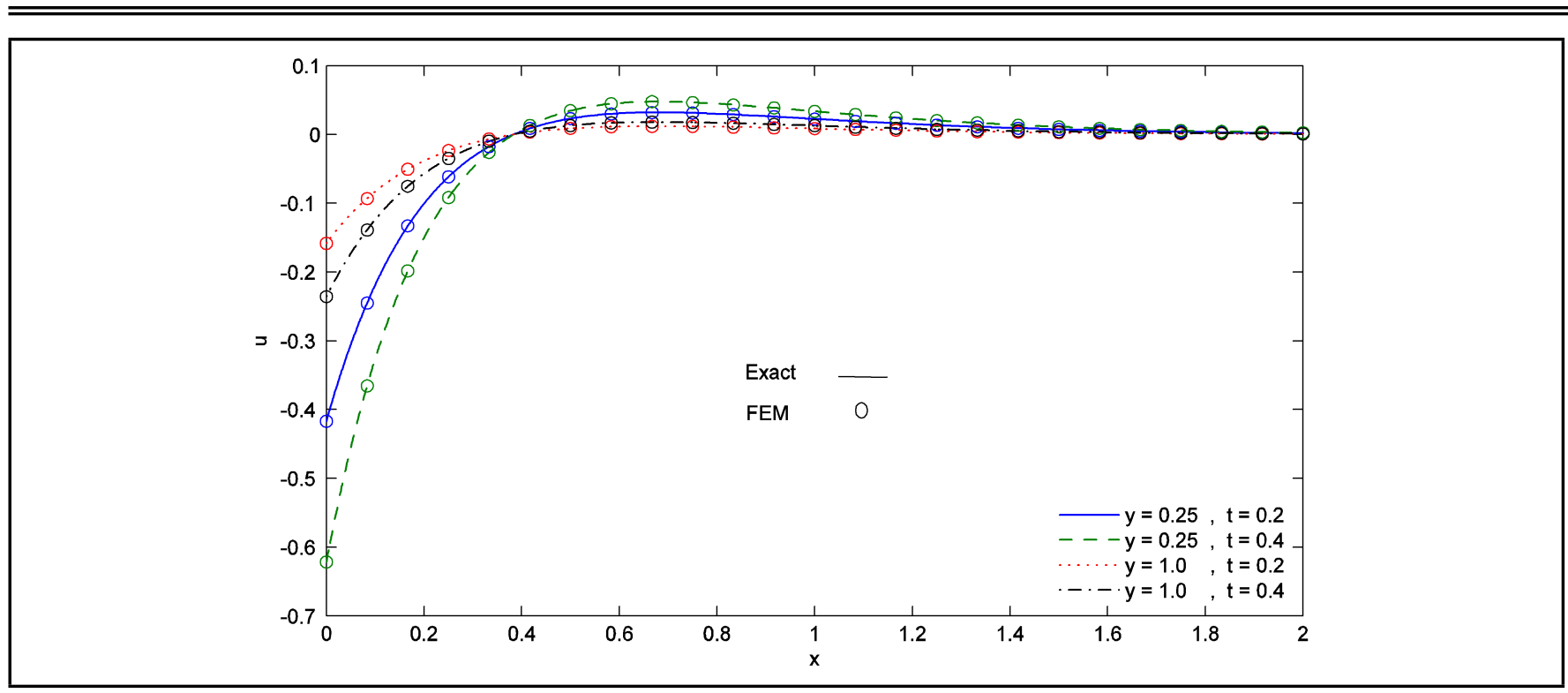

Figure 1. Horizontal displacement $u$ distribution against $x$.

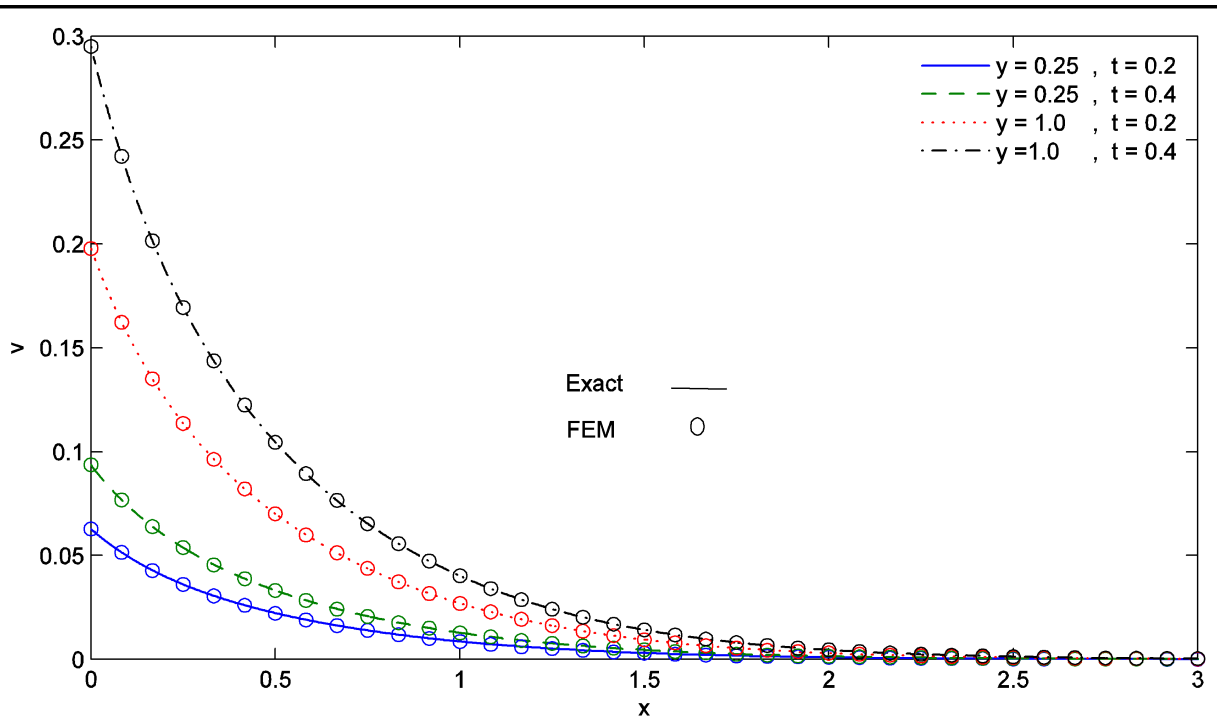

Figure 2. Vertical displacement $v$ distribution against $x$.

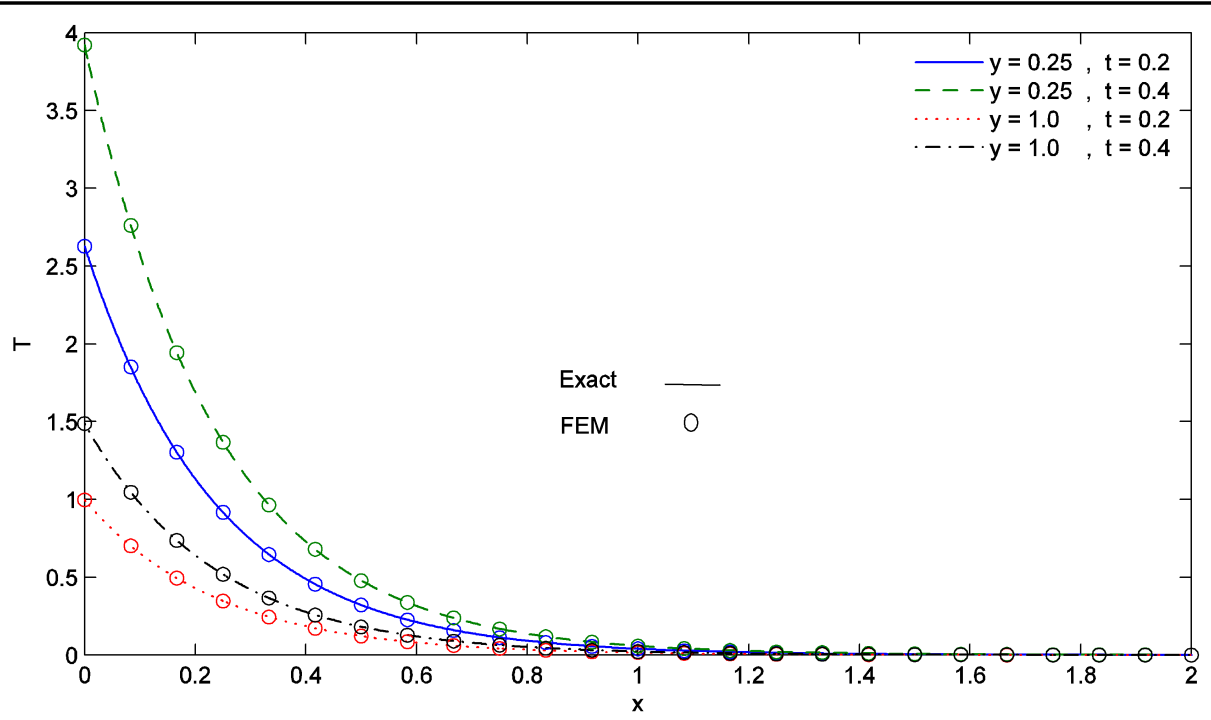

Figure 3. Temperature distribution against $x$. 


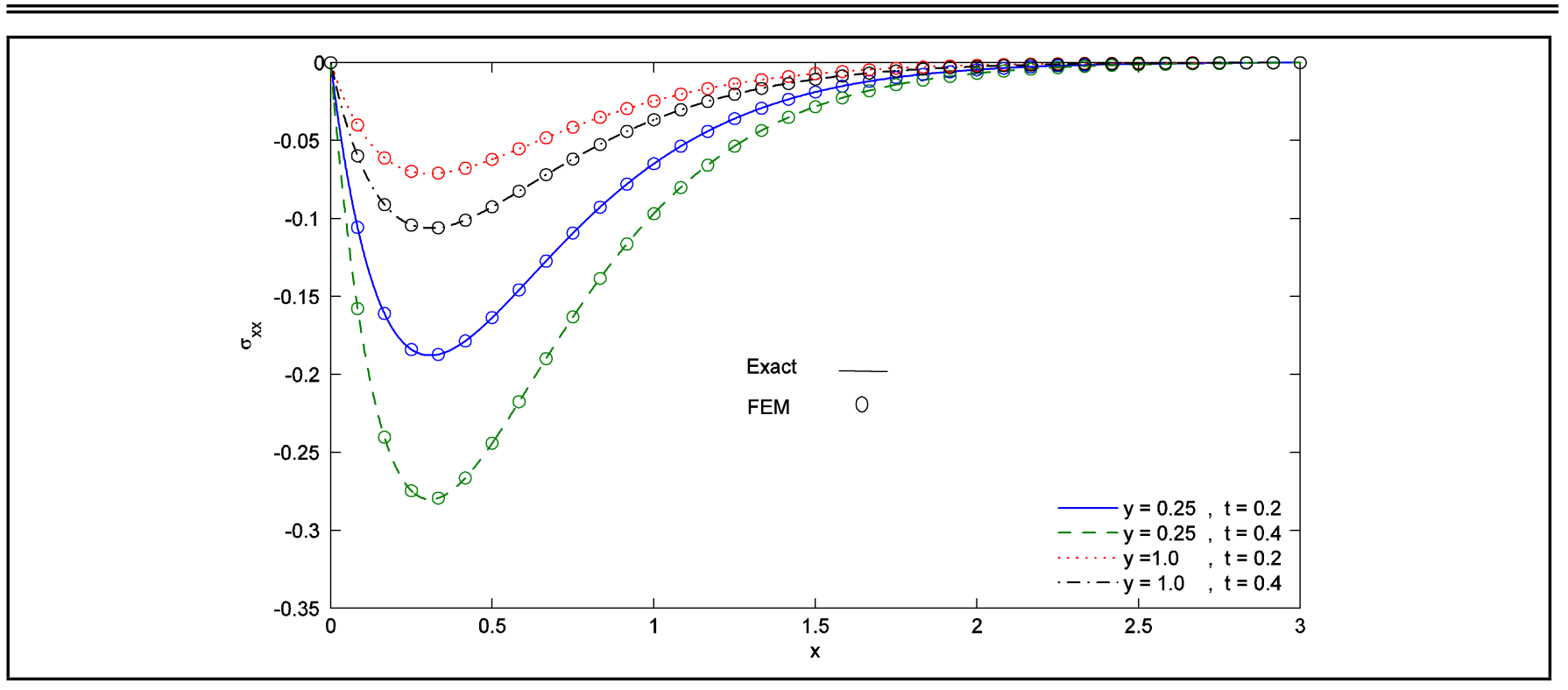

Figure 4. Stress component $\sigma_{x x}$ distribution against $x$.

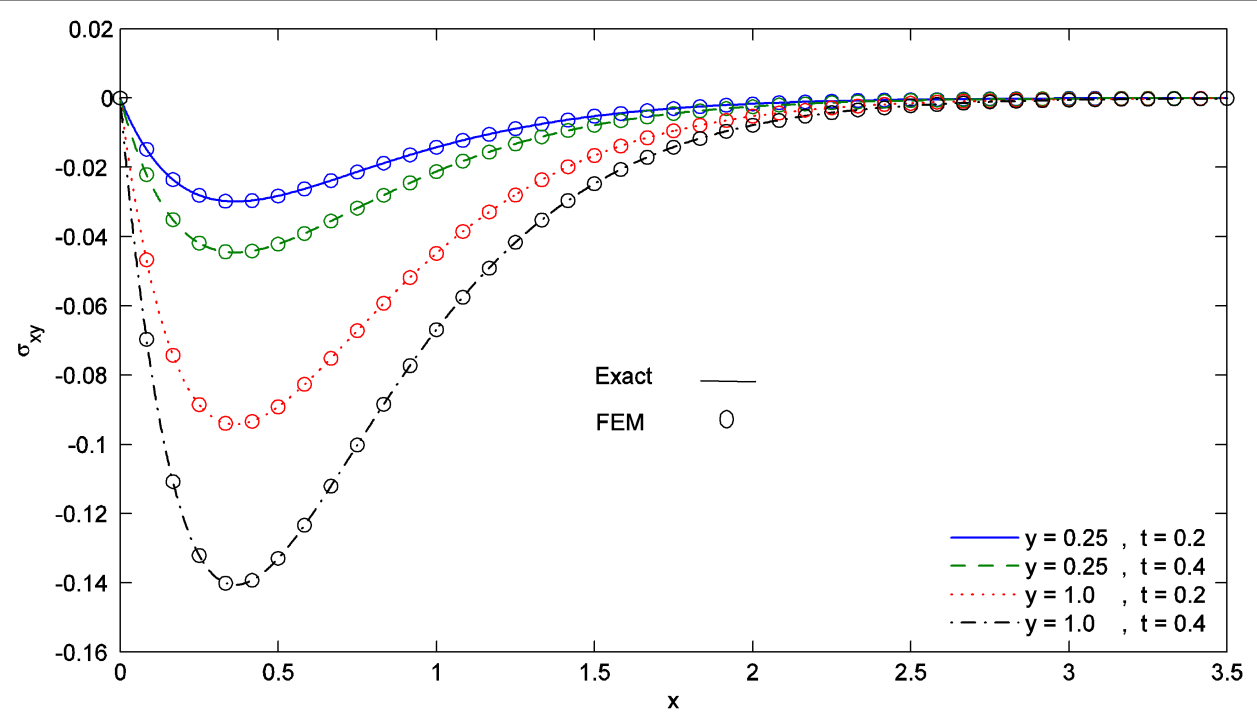

Figure 5. Stress component $\sigma_{x y}$ distribution against $x$.

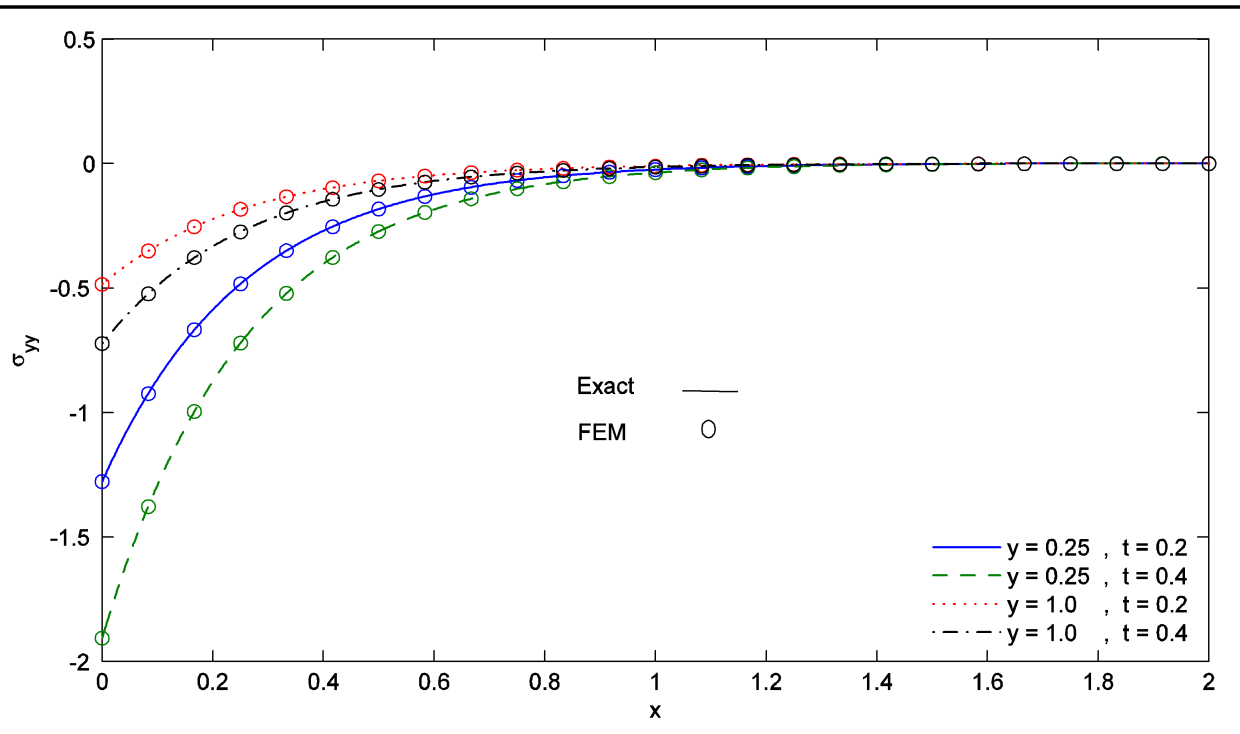

Figure 6. Stress component $\sigma_{y y}$ distribution against $x$. 
Table 1. The exact solution and finite element solution for displacement $u$.

\begin{tabular}{|c|c|c|c|}
\hline$r$ & $u_{\text {FEM }}$ & $u_{\text {Exact }}$ & Error \\
\hline 0 & -0.235886897824598 & -0.235886897826063 & $1 \times 10^{-12}$ \\
0.5 & 0.013120512987236 & 0.013120512980234 & $7 \times 10^{-12}$ \\
1 & 0.012797547589658 & 0.012797547579440 & $1 \times 10^{-11}$ \\
1.5 & 0.004223521696627 & 0.004223521719613 & $2.3 \times 10^{-11}$ \\
2 & 0.001046901631478 & 0.001046901904017 & $2.73 \times 10^{-10}$ \\
2.5 & 0.000199371868538 & 0.000199373395902 & $1.53 \times 10^{-9}$ \\
3 & 0.000020036883237 & 0.000020043693174 & $6.81 \times 10^{-9}$ \\
\hline
\end{tabular}

Table 2. The exact solution and finite element solution for displacement $v$.

\begin{tabular}{|c|c|c|c|}
\hline$r$ & $v_{\text {FEM }}$ & $v_{\text {Exact }}$ & Error \\
\hline 0 & 0.294983670973458 & 0.294983670986315 & $1 \times 10^{-11}$ \\
0.5 & 0.104525746434544 & 0.104525746427700 & $1 \times 10^{-11}$ \\
1 & 0.040208619765688 & 0.040208619670262 & $1 \times 10^{-10}$ \\
1.5 & 0.014144501531332 & 0.014144501082401 & $4.5 \times 10^{-10}$ \\
2 & 0.004482268212596 & 0.004482266450639 & $1.76 \times 10^{-9}$ \\
2.5 & 0.001285124689363 & 0.001285118516080 & $6.17 \times 10^{-9}$ \\
3 & 0.000331795331734 & 0.000331775990012 & $1.93 \times 10^{-8}$ \\
\hline
\end{tabular}

Table 3. The exact solution and finite element solution for temperature $T$.

\begin{tabular}{|c|c|c|c||}
\hline$r$ & $T_{\text {FEM }}$ & $T_{\text {Exact }}$ & Error \\
\hline 0 & 1.487547845376875 & 1.487547845377607 & $7 \times 10^{-13}$ \\
0.5 & 0.181738551772699 & 0.181738551773231 & $5 \times 10^{-13}$ \\
1 & 0.022498632162006 & 0.022498632162409 & $4 \times 10^{-13}$ \\
1.5 & 0.002847962743369 & 0.002847962742086 & $1.3 \times 10^{-12}$ \\
2 & 0.000370359458523 & 0.000370359445057 & $1.35 \times 10^{-11}$ \\
2.5 & 0.000048439450595 & 0.000048439376541 & $7.41 \times 10^{-11}$ \\
3 & 0.000005723391540 & 0.000005723064173 & $3.274 \times 10^{-11}$ \\
\hline
\end{tabular}

\section{REFERENCES}

${ }^{1}$ Chadwick, P. Thermoelasticity. The dynamical theory, Progress in Solid Mechanics, 1, 263-328, (1960).

${ }^{2}$ Nowacki, W. Thermoelasticity, Pergamon Press, (1962).

3 Nowacki, W. Dynamic Problems of Thermoelasticity, Noordhoff, Leyden, (1975).

${ }^{4}$ Lord, H. and Shulman, Y. A generalized dynamical theory of thermoelasticity, Journal of the Mechanics and Physics of Solids, 15 (5), 299-309, (1967). https://dx.doi.org/10.1016/0022-5096(67)90024-5

${ }^{5}$ Green, A. E. and Lindsay, K. A. Thermoelasticity, Journal of Elasticity, 2 (1), 1-7, (1972). https://dx.doi.org/10.1007/BF00045689

6 Verma, K. L. and Hasebe, N. Wave propagation in transversely isotropic plates in generalized thermoelasticity, Archive of Applied Mechanics, 72 (6-7), 470-482 (2002). https://dx.doi.org/10.1007/s00419-002-0215-z

7 Othman, M. I. A. Lord-Shulman theory under the dependence of the modulus of elasticity on the reference temperature in two dimensional generalized thermoelasticity, Journal of Thermal Stresses, 25 (11), 1027-1045, (2002). https://dx.doi.org/10.1080/01495730290074621

${ }^{8}$ Chandrasekharaiah, D. S. Thermoelasticity with second sound: a review, Applied Mechanics Reviews, 39 (3), 355376, (1986). https://dx.doi.org/10.1115/1.3143705
${ }^{9}$ Othman, M. I. A. Generalized electromagnetothermoelastic plane waves by thermal shock problem in a finite conductivity half-space with one relaxation time, Multidisciplinary Modeling in Materials and Structures, 1 (3), 231-250, (2005). https://dx.doi.org/10.1163/157361105774538557

10 Othman, M. I. A. Effect of rotation on plane waves in generalized thermo-elasticity with two relaxation times, International Journal of Solids and Structures, 41 (11-12), 2939-2956, (2004). https://dx.doi.org/10.1016/j.jjsolstr.2004.01.009

11 Othman, M. I. A. and Song, Y. Q. The effect of rotation on the reflection of magneto-thermoelastic waves under thermoelasticity without energy dissipation, Acta Mechanica, 184 (1), 189-204, (2006). https://dx.doi.org/10.1007/s00707-006-0337-4

12 Ignaczak, J. Generalized thermoelasticity and its applications, Thermal Stresses, Vol. III, Elsevier, Oxford, (1989).

13 Ackerman, C. C. and Guyer, R. A. Temperature pulses in dielectric solids, Annals of Physics, 50 (1), 128-185, (1968). https://dx.doi.org/10.1016/0003-4916(68)90320-5

14 Jackson, H. E., Walker, C. T., and McNelly, T. F. Second sound in NaF, Physical Review Letters, 25 (1), 26-28, (1970). https://dx.doi.org/10.1103/PhysRevLett.25.26

15 Jackson, H. E. and Walker, C. T. Thermal conductivity, second sound, and phonon-phonon interactions in NaF, Physical Review B, 3 (4), 1428-1439, (1971). https://dx.doi.org/10.1103/PhysRevB.3.1428

${ }^{16}$ Rogers, S. J. Transport of heat and approach to second sound in some isotopically pure Alkali-Halide crystals, Physical Review B, 3 (4), 1440-1457, (1971). https://dx.doi.org/10.1103/PhysRevB.3.1440

17 Narayanmurti, V. and Dynes, R. C. Observation of second sound in bismuth, Physical Review Letters, 28 (22), 1461-1465, (1972). https://dx.doi.org/10.1103/PhysRevLett.28.1461

${ }^{18}$ Marin, M. Harmonic vibrations in thermoelasticity of microstretch materials, ASME Journal of Vibration and Acoustics, 132 (4), (2010). https://dx.doi.org/10.1115/1.4000971

19 Green, A. E. and Naghdi, P. M. A re-examination of the basic postulates of thermomechanics, Proceedings: Mathematical and Physical Sciences, 432 (1885), 171-194, (1991).

${ }^{20}$ Green, A. E. and Naghdi, P. M. Thermoelasticity without energy dissipation, Journal of Elasticity, 31 (3), 189-208, (1993). https://dx.doi.org/10.1007/BF00044969

${ }^{21}$ Green, A. E. and Naghdi, P. M. On undamped heat waves in an elastic solid, Journal of Thermal Stresses, 15 (2), 252-264, (1992). https://dx.doi.org/10.1080/01495739208946136 
22 Chandrasekharaiah, D. S. A note on the uniqueness of solution in the linear theory of thermoelasticity without energy dissipation, Journal of Elasticity, 43 (3), 279-283, (1996). https://dx.doi.org/10.1007/BF00042504

23 Chandrasekharaiah, D. S. A uniqueness theorem in the theory of thermoelasticity without energy dissipation, Journal of Thermal Stresses, 19 (3), 267-272, (1996). https://dx.doi.org/10.1080/01495739608946173

24 Othman, M. I. A. and Atwa, S. Y. The effect of magnetic field on 2-D problem of generalized thermoelasticity with energy dissipation, International Journal of Industrial Mathematics, 3 (3), 213-226, (2011).

25 Othman, M. I. A. and Atwa, S. Y. Response of micropolar thermoelastic medium with voids due to various source under Green-Naghdi theory, Acta Mechanica Solida Sinica, 25 (2), 197-209 (2012). https://dx.doi.org/10.1016/S08949166(12)60020-2

26 Roychoudhuri, S. K. and Dutta, P. S. Thermoelastic interaction without energy dissipation in an infinite solid with distributed periodically varying heat sources, International Journal of Solids and Structures, 42 (14), 4192-4203, (2005). https://dx.doi.org/10.1016/j.ijsolstr.2004.12.013

27 Banik, S., Mallik, S. H., and Kanoria, M. Thermoelastic interaction with energy dissipation in an infinite solid with distributed periodically varying heat sources, International Journal of Pure and Applied Mathematics, 34 (2), 231-245, (2007).

28 Othman, M. I. A. and Atwa, S. Y. Thermoelastic plane waves for an elastic solid half-space under hydrostatic initial stress of type III, Meccanica, 47 (6), 1337-1347, (2012). https://dx.doi.org/10.1007/s11012-011-9517-y

29 Zienkiewicz, O. C. and Taylor, R. L. The Finite Element Method, 5th ed., Vol 3: Fluid dynamics, ButterworthHeinemann, (2000).

30 Abbas, I. A. and Youssef, H. M. Finite element analysis of two-temperature generalized magneto-thermoelasticity, Archive of Applied Mechanics, 79 (10), 917-925, (2009). https://dx.doi.org/10.1007/s00419-008-0259-9

31 Abbas, I. A. Generalized magneto-thermoelasticity in a non-homogeneous isotropic hollow cylinder using finite element method, Archive of Applied Mechanics, 79 (1), 4150, (2009). https://dx.doi.org/10.1007/s00419-008-0206-9

32 Abbas, I. A. and Abd-alla, A. N. Effects of thermal relaxations on thermoelastic interactions in an infinite orthotropic elastic medium with a cylindrical cavity, Archive of Applied Mechanics, 78 (4), 283-293, (2008). https://dx.doi.org/10.1007/s00419-007-0156-7

33 Abbas, I. A. and Othman, M. I. A. Generalized thermoelasticity of thermal shock problem in an isotropic hollow cylinder and temperature dependent elastic moduli, Chinese Physics B, 21 (1), (2012). https://dx.doi.org/10.1088/1674$1056 / 21 / 1 / 014601$
34 Abbas, I. A. Generalized magneto-thermoelastic interaction in a fiber-reinforced anisotropic hollow cylinder, International Journal of Thermophysics, 33 (3), 567-579, (2012). https://dx.doi.org/10.1007/s10765-012-1178-0

35 Abbas, I. A. A GN model for thermoelastic interaction in an unbounded fiber-reinforced anisotropic medium with a circular hole, Applied Mathematics Letters, 26 (2), 232239, (2013). https://dx.doi.org/10.1016/j.aml.2012.09.001

36 Othman, M. I. A. and Abbas, I. A. Generalized thermoelasticity of thermal shock problem in a non-homogeneous isotropic hollow cylinder with energy dissipation, International Journal of Thermophysics, 33 (5), 913-923, (2012). https://dx.doi.org/10.1007/s10765-012-1202-4

37 Abbas, I. A., Kumar, R., and Chawla, V., Response of thermal source in a transversely isotropic thermoelastic half-space with mass diffusion by finite element method, Chinese Physics B, 21 (8), (2012). https://dx.doi.org/10.1088/1674-1056/21/8/084601

38 Abbas, I. A. and Othman, M. I. A. Plane waves in generalized thermo-micro-stretch elastic solid with thermal relaxation using finite element method, International Journal of Thermophysics, 33 (12), 2407-2423, (2012). https://dx.doi.org/10.1007/s10765-012-1340-8

39 Zenkour, A. M. and Abbas, I. A. Electro-magnetothermoelastic response of infinite functionally graded cylinders without energy dissipation, Journal of Magnetism and Magnetic Materials, 395, 123-129, (2015). https://dx.doi.org/10.1016/j.jmmm.2015.07.038

40 Das, N. C., Lahiri, A., and Sarkar, S. Eigenvalue approach three dimensional coupled thermoelasticity in a rotating transversely isotropic medium, Tamsui Oxford Journal of Mathematical Sciences, 25 (3), 237-257, (2009).

41 Abbas, I. A. and Youssef, H. M. Two-dimensional fractional order generalized thermoelastic porous material, Latin American Journal of Solids and Structures, 12 (7), 1415-1431, (2015). https://dx.doi.org/10.1590/167978251584

42 Abbas, I. A. Eigenvalue approach to fractional order generalized magneto-thermoelastic medium subjected to moving heat source, Journal of Magnetism and Magnetic Materials, 377, 452-459, (2015). https://dx.doi.org/10.1016/j.jmmm.2014.10.159

43 Abbas, I. A. Eigenvalue approach in a three-dimensional generalized thermoelastic interactions with temperaturedependent material properties, Computers and Mathematics with Applications, 68 (12), 2036-2056, (2014). https://dx.doi.org/10.1016/j.camwa.2014.09.016

44 Dhaliwal, R. S. and Singh, A. Dynamic Coupled Thermoelasticity, Hindustan Publishing Corporation, Delhi, (1980). 